\title{
Early Detection Research Network
}

National Cancer Institute

\section{Source}

National Cancer Institute. Early Detection Research Network. NCI Thesaurus. Code C19265.

Established by $\mathrm{NCl}$ to identify and evaluate molecular biomarkers and technologies for earlier detection and risk assessment of all major cancers. The EDRN is a national network of academic and industry investigators with expertise in laboratory and clinical sciences, biostatistics, informatics, and public health. 